\title{
Manipulating miR-125a-5p to regulate cancer stem cells phenotype and epithelial to mesenchymal transition in glioblastoma
}

\author{
Yeqin Sha \\ Daoyun Lei \\ D Lianping $\mathrm{He}^{2}$
}

1. Jiangsu University, Jiangsu, Zhenjiang 212013 , China. 2. Department of Immunology, Nanjing medical university, Nanjing, Jiangsu 211166, China.

Yeqin Sha and Daoyun Lei contributed to this work.

http://dx.doi.org/10.1590/1806-9282.66.5.706

Dear Editor,

We read the study by Zhu X-D et al. ${ }^{1}$ with great interest. The study demonstrated that miR-125a-5p could inhibit cancer stem cell phenotype and epithelial to mesenchymal transition in glioblastoma. The author concluded that miR-125a-5p might be a novel therapy target for glioblastoma. This study disclosed the involvement of miRNA in the progression of glioblastoma, providing potential approaches for glioblastoma treatment and prevention. Considering the high prevalence and lethality of glioblastoma in the population, it is of great clinical significance to explore novel therapeutic targets for glioblastoma treatment. However, in our opinion, more studies should be conducted so that the conclusion could be more convincing.

To begin with, different study groups have identified that lots of miRNAs play a vital role in glioblastoma pathogenesis ${ }^{2}$ and epithelial to mesenchymal transition $^{3}$. Therefore, the bioinformatics method is a better way of finding different expressions of miRNAs between glioblastoma tissues and adjacent normal tissues. Additionally, the results of scratch wound-healing motility assays and transwell migration assays should be displayed to confirm that miR-125a-5p may suppress migration and invasion of glioblastoma.

Although a large number of studies have revealed that miRNAs have different functions in the pathogenesis of various diseases, few miRNAs have been actually applied as a therapy target. The main advantage of the use of miRNAs as a therapy target is that they might influence different physiological and pathological conditions, including chronic inflammation and other non-tumor pathologies ${ }^{4}$. Therefore, further animal studies should be conducted to confirm the overall effect of miR-125a-5p on epithelial to mesenchymal transition in glioblastoma.

\section{REFERENCES}

1. Zhu X-D, Gao Z-J, Pang Q, Zheng G-D. miR-125a-5p inhibits cancer stem cells phenotype and epithelial to mesenchymal transition in glioblastoma. Rev Assoc Med Bras. 2020;66(4);445-451.

2. Ahir BK, Ozer H, Engelhard HH, Lakka SS. MicroRNAs in glioblastoma pathogenesis and therapy: a comprehensive review. Crit Rev Oncol Hematol. 2017;120:22-33.

3. McCubrey JA, Fitzgerald TL, Yang LV, Lertpiriyapong K, Steelman LS, Abrams $\mathrm{SL}$, et al. Roles of GSK-3 and microRNAs on epithelial mesenchymal transition and cancer stem cells. Oncotarget. 2017;8(8):14221-50.

4. Wang H, Peng R, Wang J, Qin Z, Xue L. Circulating microRNAs as potential cancer biomarkers: the advantage and disadvantage. Clin Epigenetics. 2018;10:59. 